\title{
The DUNE Near Detector
}

\section{A. Mastbaum ${ }^{a, 1, *}$}

${ }^{a}$ Department of Physics and Astronomy, Rutgers University, Piscataway, NJ 08854, USA

E-mail: mastbaum@physics.rutgers.edu

The Deep Underground Neutrino Experiment (DUNE) is an upcoming long-baseline neutrino experiment which will study neutrino oscillations. Neutrino oscillations will be detected at the DUNE Far Detector $1300 \mathrm{~km}$ away from the start of the beam at Fermilab. The DUNE Near Detector (ND) will be located on-site at Fermilab, and will be used to provide an initial characterization of the neutrino beam, as well as to constrain systematic uncertainties on neutrino oscillation measurements. The detector suite consists of a modular LArTPC (ND-LAr), a magnetized gaseous argon time projection chamber (ND-GAr) surrounded by an electromagnetic calorimeter, and the System for on-Axis Neutrino Detection (SAND), composed of a magnetized electromagnetic calorimeter and inner tracker. In these proceedings, these detectors and their physics goals will be discussed.

*** The European Physical Society Conference on High Energy Physics (EPS-HEP2021), ***

*** 26-30 July $2021 * * *$

*** Online conference, jointly organized by Universität Hamburg and the research center DESY ***

${ }^{1}$ For the DUNE Collaboration

* Speaker 


\section{Introduction}

The upcoming Deep Underground Neutrino Experiment (DUNE) will explore long-baseline neutrino oscillation physics as part of a portfolio of measurements. The oscillation physics program is centered on a measurement of any $C P$ violation in neutrino oscillations, potentially connected to the matter-antimatter asymmetry observed in our universe, and will also yield precise measurements of mixing angles and the neutrino mass ordering while providing a strict test of the three-neutrino oscillation model with both appearance and disappearance measurements in neutrino and antineutrino beams [1]. These measurements are part of a broader physics program including sensitivity to neutrino astrophysics signals such as supernovae [2] and solar neutrinos [3], nucleon decay, and a wide array of searches for Beyond the Standard Model (BSM) physics [4].

The experiment will be comprised of a Near and Far Detector complex, with the Far Detector (FD) [3] located deep underground at a baseline of $1300 \mathrm{~km}$, and consisting of multiple large-scale liquid argon time projection chamber (LArTPC) modules. Four caverns each capable of holding a 17 kton LArTPC module enable a DUNE FD with up to 68 kton of LAr target mass. A broadband neutrino beam at Fermilab, with power upgradaeble to $2.4 \mathrm{MW}$, will provide an intense source of primarily muon neutrinos or muon antineutrinos for DUNE, with the sign selected via the beam focusing configuration. The DUNE Near Detector (ND) complex [5] will be located at Fermilab, close to the point of neutrino production. Situated in this intense neutrino flux, the ND complex will provide a high-statistics measurement of neutrino interactions in the initial, unoscillated beam. This will enable characterization of the neutrino beam free from the impact from oscillations and provide powerful constraints on systematic uncertainties for neutrino oscillation physics via precise measurements of the beam flux and neutrino interactions. The detector suite will include a LArTPC and a magnetized gaseous argon TPC with an electromagnetic calorimeter (ECAL), where both systems can move several meters transverse to the beam. An additional on-axis detector will provide continuous beam monitoring. Each of these systems is described in further detail in Section 2.

\section{The DUNE Near Detector Complex}

The DUNE Near Detector (ND) complex will be located at Fermilab, close to the point of neutrino production at a baseline of approximately $500 \mathrm{~m}$ from the proton beam target in a new underground facility at a depth of about $60 \mathrm{~m}$. It will combine multiple complementary detector technologies to provide a large-statistics sample of neutrino interactions with which to constrain flux- and cross section-related systematic uncertainties in oscillation measurements based on a near/far comparison, in addition to an independent program measuring neutrino interactions and conducting searches for Beyond the Standard Model (BSM) physics. Figure 1 shows the overall design, and the main ND components are described briefly in the following subsections.

\subsection{ND-LAr}

The Liquid Argon Near Detector, ND-LAr, is a liquid argon time projection chamber (LArTPC) using the same liquid argon (LAr) target and detector technology as is used in the DUNE Far Detector LArTPCs. This will provide valuable information in a near-far comparison, since neutrino interactions and the propagation of final state particles in the bulk detector material are identical, 


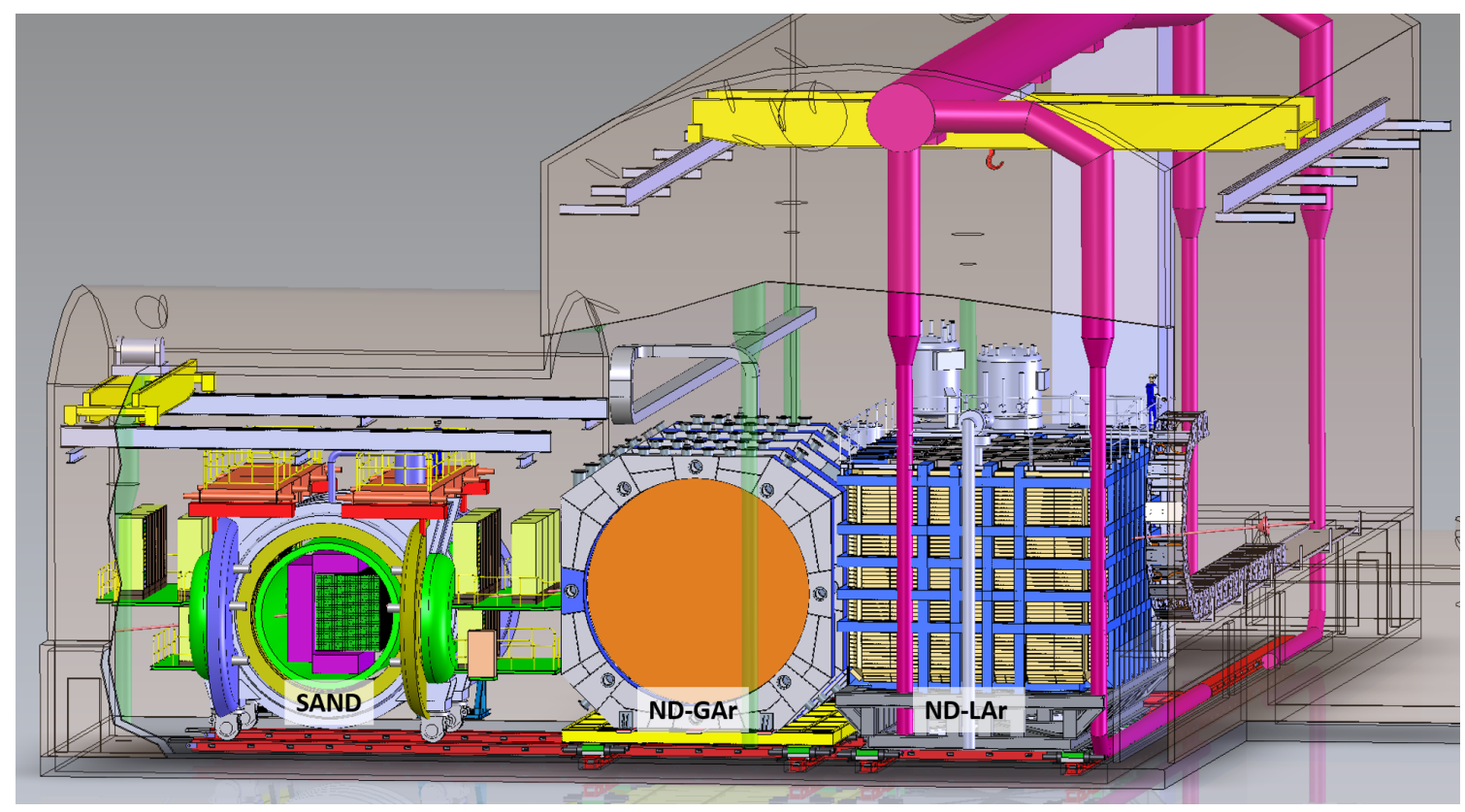

Figure 1: A rendering of the DUNE Near Detector hall, with the neutrino beam moving from right to left and slightly downward through the detector centers. The detectors include (from right) the ND-LAr (blue), ND-GAr (orange/grey), and SAND (green/purple). Also visible are the tracks used to move the ND-LAr and ND-GAr, per the DUNE-PRISM off-axis movement system. Figure from Ref. [5].

and the detector response and reconstruction techniques are similar. Additionally, the high density of the LAr target, combined with the intense flux at the near site, will provide very high statistics event samples, with tens of neutrino interactions present within the detector's active region in each pulse (spill) of the neutrino beam.

It is necessary to uniquely associate deposited energy with a single neutrino interaction in this high-event multiplicity environment, motivating a modular design for ND-LAr. Traditionally, LArTPCs have been constructed with large monolithic LAr volumes, instrumented with a TPC to measure ionization charge and an array of photon detectors to measure the scintillation light. Correctly associating these two signals is essential, as the prompt scintillation light is used to resolve the inherent ambiguity in TPC readout between the time that charge was deposited and its location along the drift direction. To mitigate matching ambiguities in high-pileup events, the ND-LAr will be constructed from a $7 \times 5$ array of $1 \times 1 \times 3 \mathrm{~m}^{3}$ detector modules, each containing two optically isolated TPCs with a $50 \mathrm{~cm}$ drift length.

A cutaway view of a module is shown in Figure 2a. Visible are the high-coverage light detection system, low-profile field cage constructed from a resistive polymer material, and the LArPix [6] charge readout system. The LArPix system uses a 2D array of charge-sensitive 'pixel' PCB pads read out by a custom mixed-signal ASIC to provide direct-to-3D charge location information, without requiring matching of charge across wire planes. This further reduces pileup-related ambiguities, as charge position reconstruction is not dependent on resolving overlaps in $2 \mathrm{D}$ projections.

The ND-LAr will collect a high-statistics, high-precision sample of neutrino interactions in LAr. For example, while taking data in the beam configuration where primarily $v_{\mu}$ are produced, 


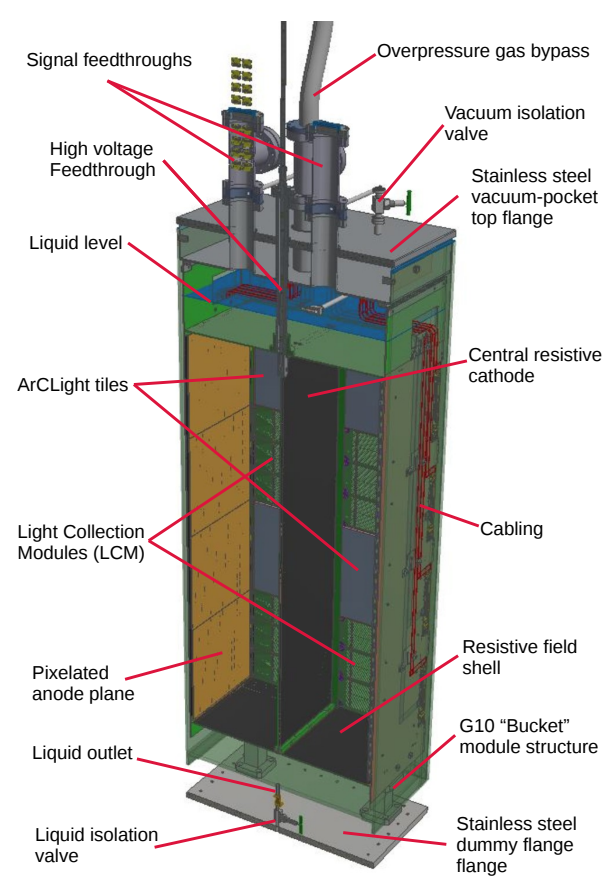

(a) Cutaway of a single ND-LAr module, showing the two TPC drift volumes and other major components.

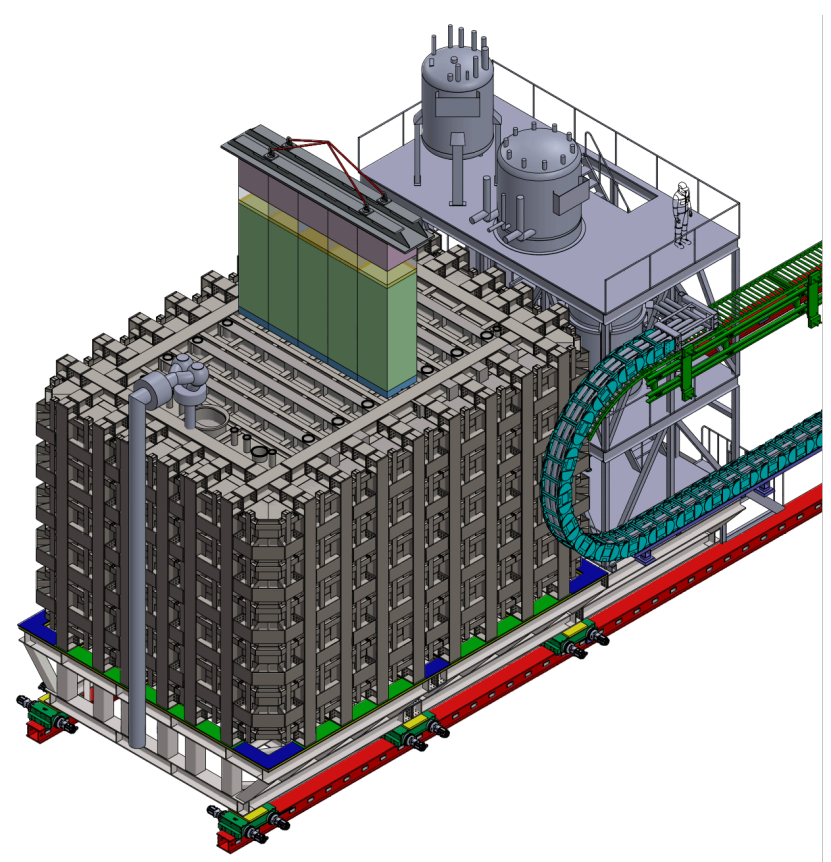

(b) The ND-LAr cryostat design, showing the constituent detector modules and system for DUNE-PRISM movement.

Figure 2: Drawings illustrating the ND-LAr detector design.

about 30 million $v_{\mu}$ charged-current (CC) events per year are expected. The large flux and electron density additionally permit a precise direct measurement of the flux via neutrino-electron elastic scattering, thus the ND-LAr provides important constraints on both the flux and interactions in LAr supporting DUNE's physics goals.

\subsection{ND-GAr}

With DUNE's multi-GeV neutrino energies and the size constraints on the ND detectors, a large fraction of the particles produced in ND-LAr, most importantly $v_{\mu} \mathrm{CC}$ muons, will exit the downstream end of that detector. In order to precisely measure the momentum of these ND-LAr exiting muons, a downstream tracking spectrometer is required. Figure $3 \mathrm{~b}$ shows the acceptance for muon tracks originating from the ND-LAr fiducial volume. While only the lowest-energy tracks are contained in ND-LAr, reconstruction using the downstream ND-GAr ECAL and gas TPC affords high and uniform acceptance across a broad energy range.

The DUNE ND-GAr will be situated immediately downstream of ND-LAr and consist of a central high pressure gaseous argon TPC (HPgTPC) surrounded by an electromagnetic calorimeter, which are both within a solenoidal magnetic field provided by a superconducting magnet, as illustrated in Figure 3a. The central detector again uses the same nuclear target as the ND-LAr and the DUNE FD, but with a lower-density medium, achievable particle tracking thresholds are substantially lower, and multiple scattering and bulk reinteractions of final state particles are minimized, providing an independent and detailed view of neutrino-argon interactions. With a 


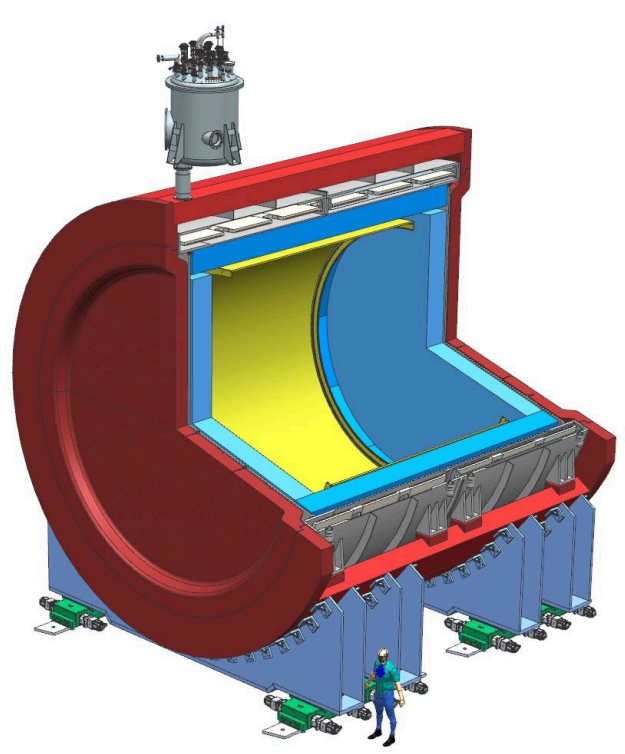

(a) Rendering of the ND-GAr design, with a cutaway showing the magnet, ECAL, and inner gaseous argon TPC.

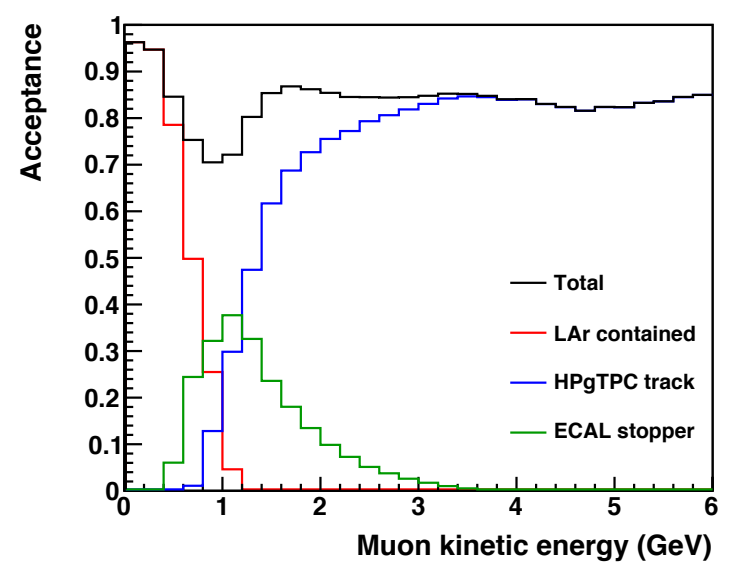

(b) Acceptance for muons originating from interactions in ND-LAr, showing the impact of ND-GAr for reconstruction of events not contained in the LAr. Figure from Ref. [5].

Figure 3: Figures illustrating the ND-GAr detector design.

magnetic field, the ND-GAr tracker will provide curvature-based measurements of charged particle track momentum, and also distinguish the charge sign, including differentiating between positively and negatively charged muons produced in $v_{\mu}$ and $\bar{v}_{\mu} \mathrm{CC}$ interactions. This enables crucial constraints on the respective event rates at the FD, of particular importance for the $v_{e}$ and $\bar{v}_{e}$ appearance channels in the oscillation measurements.

The design of the ND-GAr HPgTPC is based on the ALICE TPC at CERN [7], and will indeed reuse a set of readout chambers from ALICE, which is undergoing an upgrade. In contrast to that experiment, ND-GAr will use an $\mathrm{Ar}_{-} \mathrm{CH}_{4}$ gas mixture, at pressures up to $10 \mathrm{~atm}$ and a new readout system that may share pixel-based charge readout technologies with ND-LAr.

\subsection{DUNE-PRISM}

DUNE-PRISM is a system for off-axis movement, wherein ND-LAr and ND-GAr can travel up to $\sim 30 \mathrm{~m}$ transverse to the beam axis. This enables scanning measurements as a function of off-axis angle, which changes the neutrino flux. Relative to the broadband on-axis flux, higher off-axis angles correspond to neutrino fluxes with a lower mean energy and narrower distribution. Event samples obtained across this angular range will therefore be sensitive to different mixtures of neutrino interaction types (i.e. from rich in resonance production to quasielastic-dominated).

This serves two important purposes relevant to the near-far oscillation measurements. First, one can combine unoscillated off-axis ND samples to construct a data-driven prediction of the oscillated FD event rate, reducing model dependence in a near-far extrapolation. Additionally, the energy dependent neutrino interaction measurements will mitigate degeneracies with cross section effects, improving modeling of both the flux and interactions. Overall, the goal of the DUNE-PRISM 


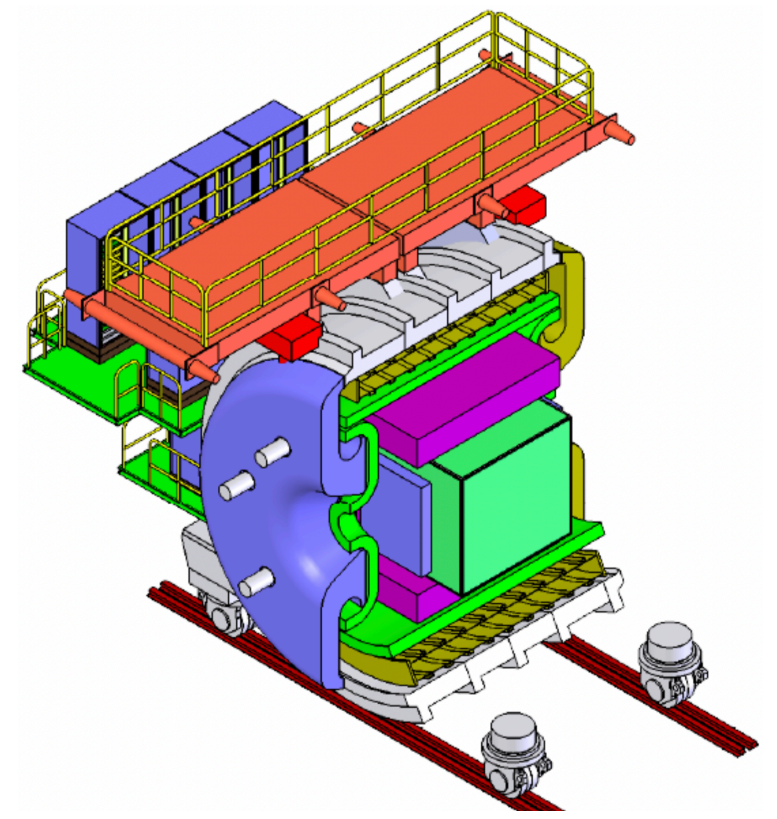

(a) Rendering of the SAND reference design, with a cutaway showing the magnet, ECAL, and tracker regions.

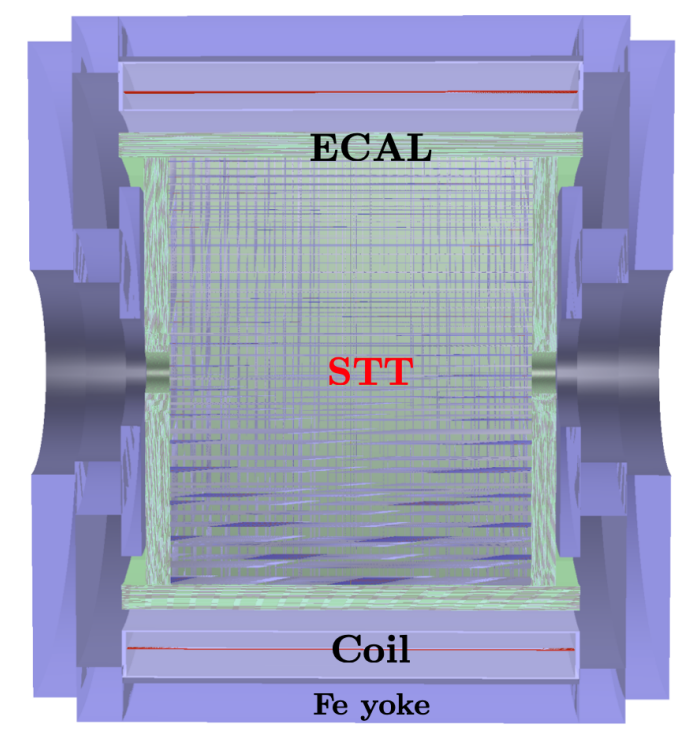

(b) Design of SAND with a Straw Tube Tracker (STT) as the inner detector.

Figure 4: Figures illustrating the SAND detector design. Figures from Ref. [5].

system is to minimize systematic uncertainties related to flux and cross section, using a data-driven approach so as not to rely on assumptions about the relevant degrees of freedom in the model.

\subsection{SAND}

SAND, the System for On-axis Neutrino Detection, will provide a fixed on-axis detector for continuous monitoring of the neutrino beam, including when the ND-LAr and ND-GAr are in off-axis positions. This is essential to ensure that changes in the event rates during DUNE-PRISM scans are truly due to the off-axis position, rather than transient or changing beam conditions.

SAND is comprised of multiple detector components, forming a composite target and tracking system including a tracker and electromagnetic calorimeter in a solenoidal magnetic field. The ECAL and magnet are derived from the KLOE experiment [8]. The tracker technology, which is being finalized, may be based on a Straw Tube Tracker (STT) or 3D scintillator tracker (3DST), together with a TPC. Figure 4 shows the reference design and the layout for the STT.

In addition to providing continuous beam monitoring, SAND is capable of a suite of highprecision interaction measurements on carbon or hydrocarbon targets that are complementary to measurements in ND-LAr and ND-GAr. A design incorporating an argon target could also provide additional $v$-Ar samples for comparison.

\section{Conclusions}

The DUNE Near Detector complex will employ multiple, complementary detector technologies, including a liquid argon TPC (ND-LAr), a magnetized gaseous argon TPC with an ECAL 
(ND-GAr), an on-axis detector system (SAND), and the capability for off-axis movement of NDLAr and ND-GAr (DUNE-PRISM). This system has been developed to provide a suite of inputs to the DUNE physics program, including constraints on systematic uncertainties related to flux, cross sections, and detector response. Data-driven extrapolations to the FD event rates provide built-in mitigations for model shortcomings and degeneracies.

A vigorous $R \& D$ program is underway to support design efforts. Within ND-LAr, recent work includes a test of a 70\% scale ND-LAr module prototype with cosmic rays, and this is to be followed by full-scale module test and a deployment of a $2 \times 2$ array in a neutrino beam at Fermilab. Several prototypes are in operation for ND-GAr including test stands for characterizing readout chambers, and dedicated gas and high voltage tests. The SAND design is supported by beam tests of 3DST prototypes at CERN, and joint prototyping efforts with the functionally similar SuperFGD system being developed for T2K upgrades.

The detector designs are reaching maturity, and the reference design presented here is documented in detail in the DUNE Near Detector Conceptual Design Report [5].

\section{References}

[1] DUNE collaboration, Long-baseline neutrino oscillation physics potential of the DUNE experiment, Eur. Phys. J. C 80 (2020) 978 [2006 . 16043].

[2] DUNE collaboration, Supernova neutrino burst detection with the Deep Underground Neutrino Experiment, Eur. Phys. J. C 81 (2021) 423 [2008.06647].

[3] DUNE collaboration, Deep Underground Neutrino Experiment (DUNE), Far Detector Technical Design Report, Volume II: DUNE Physics, 2002.03005.

[4] DUNE collaboration, Prospects for beyond the Standard Model physics searches at the Deep Underground Neutrino Experiment, Eur. Phys. J. C 81 (2021) 322 [2008. 12769].

[5] A. Abed Abud et al., Deep Underground Neutrino Experiment (DUNE) Near Detector Conceptual Design Report, Instruments 5 (2021) .

[6] D. Dwyer, M. Garcia-Sciveres, D. Gnani, C. Grace, S. Kohn, M. Kramer et al., LArPix: demonstration of low-power $3 d$ pixelated charge readout for liquid argon time projection chambers, JINST 13 (2018) P10007.

[7] J. Alme, Y. Andres, H. Appelshäuser, S. Bablok, N. Bialas, R. Bolgen et al., The ALICE TPC, a large 3-dimensional tracking device with fast readout for ultra-high multiplicity events, Nucl. Instrum. Meth. A 622 (2010) 316.

[8] K. Smith, A. Broadbent, M. Greenslade, S. Harrison, D. Jenkins, J. Ross et al., Progress in the design and manufacture of the KLOE solenoid for the DAPHNE ring at Frascati, IEEE Trans. Appl. Supercond. 7 (1997) 630. 